\title{
Une étude de productions artisanales et de leurs usages dans l'espace public et l'espace sacré d'une cité cycladique. Le cas de Ténos à l'époque archaïque et classique
}

Vicky Vlachou

\section{(2) OpenEdition Journals}

Édition électronique

URL : https://journals.openedition.org/baefe/4193

DOI : $10.4000 /$ baefe. 4193

ISSN : 2732-687X

Éditeur

ResEFE

\section{Référence électronique}

Vicky Vlachou, «Une étude de productions artisanales et de leurs usages dans l'espace public et l'espace sacré d'une cité cycladique. Le cas de Ténos à l'époque archaïque et classique » [notice archéologique], Bulletin archéologique des Écoles françaises à l'étranger [En ligne], Grèce, mis en ligne le 30 août 2021, consulté le 01 septembre 2021. URL : http://journals.openedition.org/baefe/4193 ; DOI https://doi.org/10.4000/baefe.4193

Ce document a été généré automatiquement le 1 septembre 2021.

\section{(c) $(1) \&$}

Le Bulletin archéologique des Écoles françaises à l'étranger est mise à disposition selon les termes de la Licence Creative Commons Attribution - Pas d'Utilisation Commerciale - Pas de Modification 4.0 International. 
Une étude de productions artisanales et de leurs usages dans l'espace public et l'espace sacré d'une cité cycladique. Le cas de Ténos à l'époque archaïque et classique

\author{
Vicky Vlachou
}

\title{
NOTE DE L'AUTEUR
}

Date précise de l'opération : 16-31 juillet 2020

Autorité nationale présente : Éphorie des antiquités des Cyclades

Numéro de mission : 150

Partenariats institutionnels : Université d'Athènes

Établissement éditeur : EFA

Établissements porteurs de l'opération : EFA

1 Le projet « Une étude de productions artisanales et de leurs usages dans l'espace public et l'espace sacré d'une cité cycladique. Le cas de Ténos à l'époque archaïque et classique " reprend la question de l'utilisation des espaces publics et sacrés de la cité grecque, en abordant le cas d'une cité moins connue des Cyclades, celle de Ténos. Le projet est réalisé en collaboration avec l'Université d'Athènes (fouilles en cours sous la direction de professeur émérite Nota Kourou).

2 Le premier volet de ce projet porte sur la production artisanale textile à travers les données concernant le travail du textile et leur visibilité dans le contexte archéologique. Cette étude reprend une grande quantité de pesons et de fusaïoles, de 
bobines, et encore d'autres outils liés au travail du textile provenant de trois contextes contemporains mais distincts de la cité de Xobourgo : le bâtiment E, le sanctuaire de Déméter et la nécropole (parcelle Vardalakos) ${ }^{1}$. Notre étude porte sur trois axes: 1) Vérification de la production locale des outils liés au travail du textile ;2) Retracer la répartition spatiale des pesons, des fusaïoles etc., afin de distinguer les espaces liés au travail du textile ou au dépôt secondaire de ces objets (trouvés dans des tombes de la nécropole, dans des fosses où ailleurs dans l'espace de la cité) ; 3) Proposer une lecture sociologique et économique plus approfondies du travail du textile au sein de la cité, surtout en relation avec les contextes à caractère publique, privé ou sacré à travers l'exemple de Xobourgo à Ténos. Notre méthodologie a compris la description complète de chaque individu de manière macroscopique et microscopique, afin de contrôler ces résultats contre les groups de référence de la céramique local. Par la suite, on a effectué une quantification quantitative et qualitative par contexte archéologique, afin de comparer la quantité mais également l'homogénéité technique et technologique du matériel issu des contextes différents. Afin de mieux comprendre la fonction d'un tel travail dans des différentes zones de la cité archaïque et classique il est nécessaire de s'interroger sur l'utilisation des espaces publics et sacrés et sur les activités qui s'y déroulaient, surtout à l'intérieur d'une cité de petite échelle comme celle de Xobourgo à Ténos. Il semble évident que les bâtiments étudiés pourraient avoir servi aux fonctions variées pendant l'année, qui en effet nous permet de mettre en évidence des critères écologiques, économiques et territoriales.

3 La production textile dans les sanctuaires archaïques reste une question rarement traitée pour le monde grec $^{2}$. Le matériel provenant du sanctuaire de Déméter à Xobourgo, le Thesmophorion, et daté au $v^{\mathrm{e}}$ et $\mathrm{IV}^{\mathrm{e}}$ siècles avant J.-C., permet d'aborder la question du travail du textile à l'intérieur des espaces publics et sacrés de la cité3. Selon le nombre de pesons, l'homogénéité de leur forme et leur poids, et leur disposition spatiale, il semble possible de reconstituer plus précisément l'emplacement des activités liées à l'artisanat, à l'intérieur du Thesmophorion (fig. 1 et 2). Les données de Xobourgo semblent comparables aux sanctuaires fouillés surtout en Italie. L'emplacement du travail du textile au sein d'un sanctuaire a été discuté pour les sanctuaires de la Déesse à Este (Padoue), de Timpone della Motta en Calabre et d'Héra à Paestum ${ }^{4}$. La grande quantité de poids de métiers à tisser, ainsi que d'autres outils de filage et de tissage, concentrée dans des espaces bien déterminés de chaque sanctuaire, permet la reconstruction des étapes successives de la préparation des étoffes. À Ténos, les activités liées au travail du textile semblent avoir été menées à la fois dans le sanctuaire de Déméter, le Thesmophorion et dans le bâtiment $\mathrm{E}$ de la cité archaïque et classique, à caractère en partie publique et en partie privé. La présence des grands vases de stockage à l'intérieur du bâtiment $\mathrm{E}$ et la grande quantité des pesons assemblée à l'intérieur de l'espace 2 du bâtiment, soulignent davantage l'aspect multifonctionnel des espaces différents du bâtiment $\mathrm{E}$, qui semblent avoir servi des fonctions différentes et sans doute complémentaires (fig. 3 et 4). D'après l'exemple de Xobourgo, il est possible de placer les activités étroitement liées au monde féminin pendant l'antiquité, comme le filage et le tissage, au centre de la vie publique et sacrée de la cité de Ténos. En dehors d'un schéma exclusivement lié aux femmes comme donatrices des textiles, notre approche considère le travail des femmes et leurs rôles dans la vie économique de la cité de Ténos tout comme la manière dont les différents espaces de la cité pouvaient intégrer les activités liées à l'artisanat. En plus, le travail 
du textile semble avoir fait partie de l'éducation des jeunes filles de la cité et en même temps représente une expression rituelle collective lors de fêtes religieuses ${ }^{5}$.

Fig. 1. Ténos, Xobourgo. Thesmophorion.

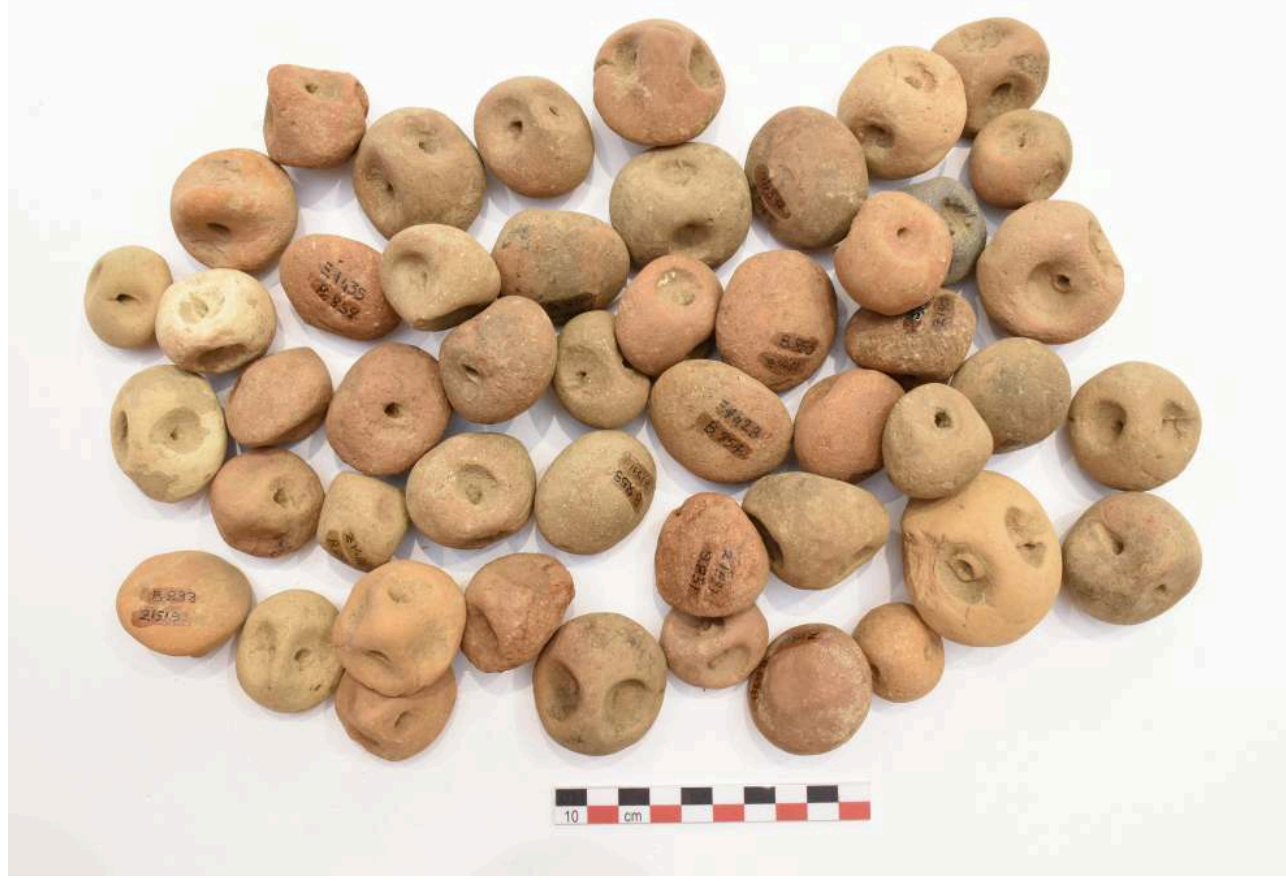

Pesons en terre cuite.

Photo de l'auteur.

Fig. 2. Ténos, Xobourgo. Thesmophorion.

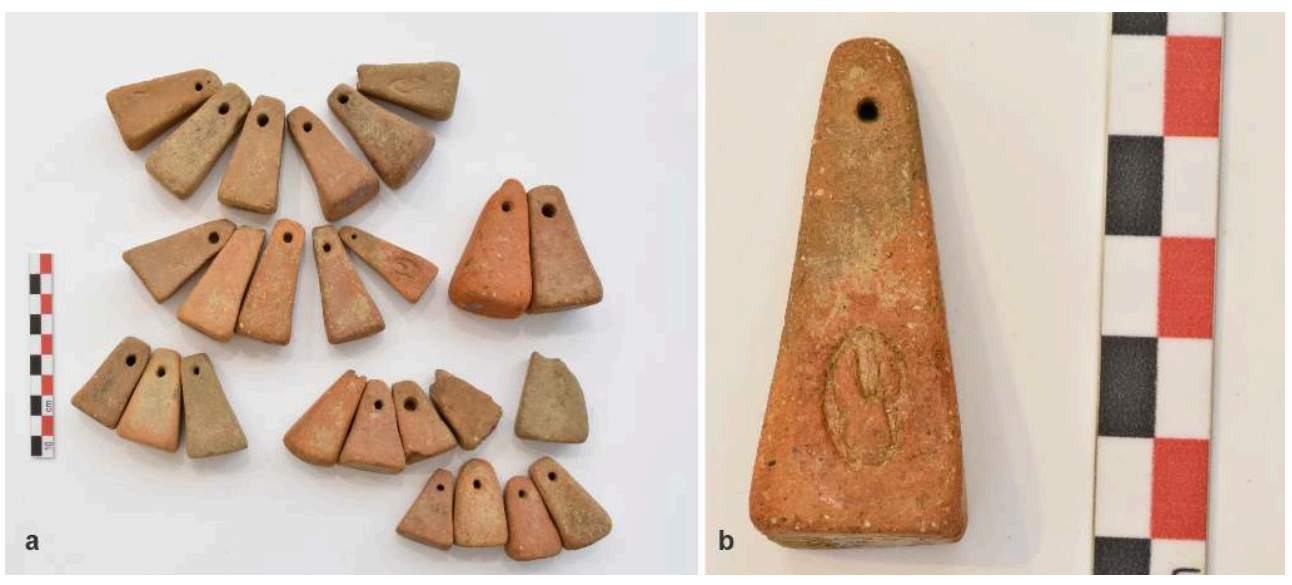

a) Pesons pyramidaux en terre cuite

b) peson avec une estampille représentante un peson pyramidal ailé.

Photos de l'auteur. 
Fig. 3. Ténos, Xobourgo. Bâtiment E.

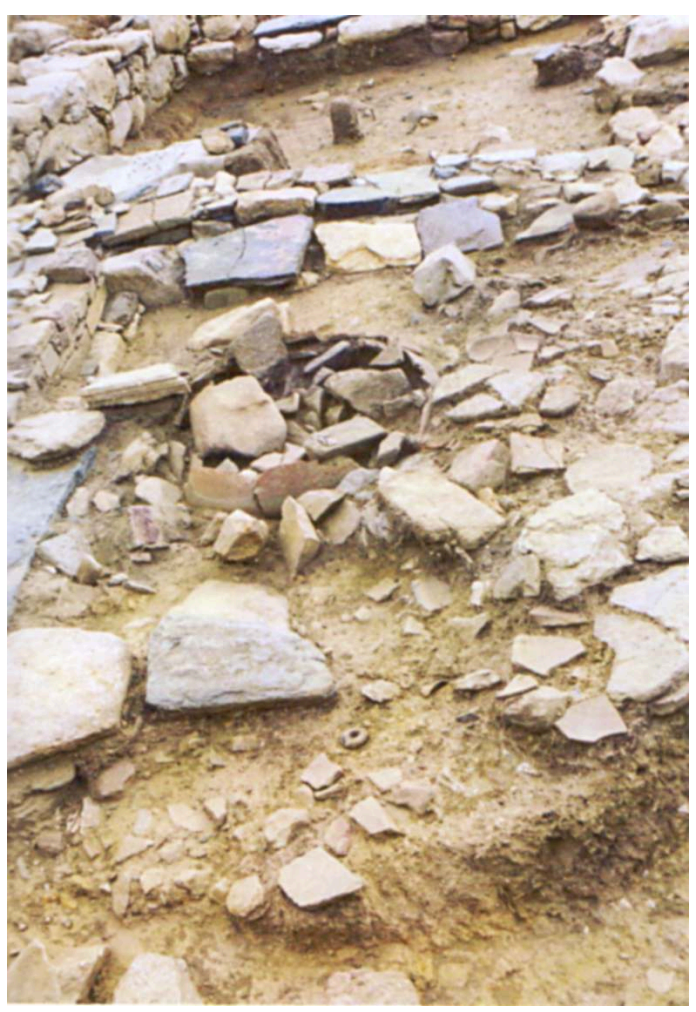

Cliché de la couche de destruction à l'intérieur de l'espace II.

D'après, ÉTIENNE, KOUROU, SIMANTONI-BOURNIA 2013, p. 79 fig. 55. 
Fig. 4a-b. Ténos, Xobourgo. Bâtiment E.

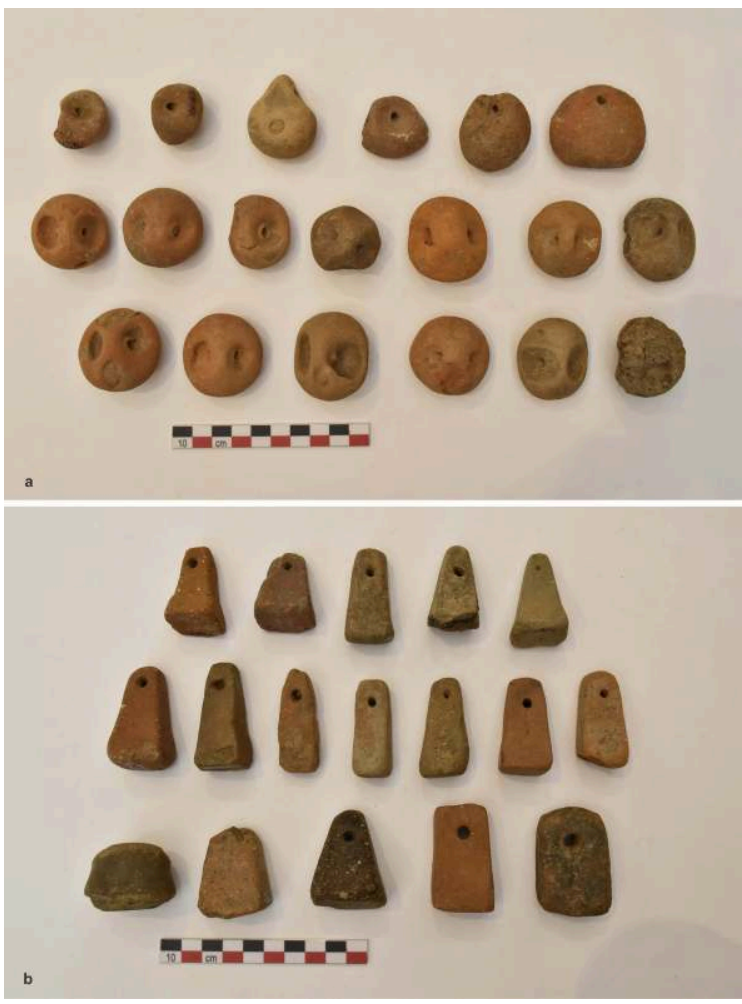

Sélection de pesons en terre cuite trouvés à l'intérieur de l'espace II.

Photo de l'auteur.

4 Le deuxième volet de notre travail porte sur les productions locales et les usages de la céramique de cuisine au sein de deux zones distinctes de la cité : le bâtiment $\mathrm{E}$ et la nécropole (parcelle Vardalakos). Les résultats de l'étude céramique du mobilier sortis lors des fouilles de la nécropole entre 2013 et 2019 constituent une première approche de la céramique consommée sur le site pendant les rituels funéraires. La méthodologie est comparable à celle poursuivie dans le cas des outils de la production textile : 1) Description des caractéristiques de la céramique culinaire de production locale à travers une étude macroscopique et microscopique, en mettant en évidence des similitudes avec d'autres productions céramiques (grands vases à stockage, pesons et fusaïoles, terres cuites) trouvées sur le site ; 2) Retracer la répartition spatiale de la céramique culinaire dans la zone nord de la nécropole en relation avec les constructions en schiste. 3) Comparer l'ensemble de la céramique culinaire trouvé dans la zone de la nécropole aux assemblages céramiques provenant du bâtiment $\mathrm{E}$, afin de tirer des conclusions plus générales sur les usages de la céramique entre les différents contextes archéologiques et sociaux.

Les formes communes, telles que les différents types de marmites (lopas, chytra) utilisées pour faire bouillir et servir des produits alimentaires (fig. 5 et 6), le matériel mobile de cuisson, comme l'eschara, employé pour griller les aliments, et des formes locales, telles que des bols de types et de dimensions divers, forment un ensemble homogène employé pendant les rites funéraires à Xobourgo. Ce type de céramique était déposé et parfois intentionnellement détruit sur les constructions en schiste de la nécropole, qui semblent avoir servi de tables d'offrandes (fig. 7). Cet ensemble constitue un cas privilégié et unique pour étudier le rôle des récipients céramiques 
dans la séquence rituelle, un sujet rarement développé dans la littérature récente. Au contraire aux enclos funéraires de type attique, les constructions en schiste de Xobourgo sont placées à proximité des tombes mais jamais en relation directe avec elles. Ainsi, nous pouvons clairement distinguer deux zones d'activité dans la nécropole; une au sud où se trouvent les tombes, et une vers le nord avec l'emplacement des constructions en schiste, des stèles funéraires et d'un bâtiment, d'une fonction probablement liée aux rituels funéraires.

Fig. 5. Ténos, Xobourgo. Nécropole (parcelle Vardalakos).

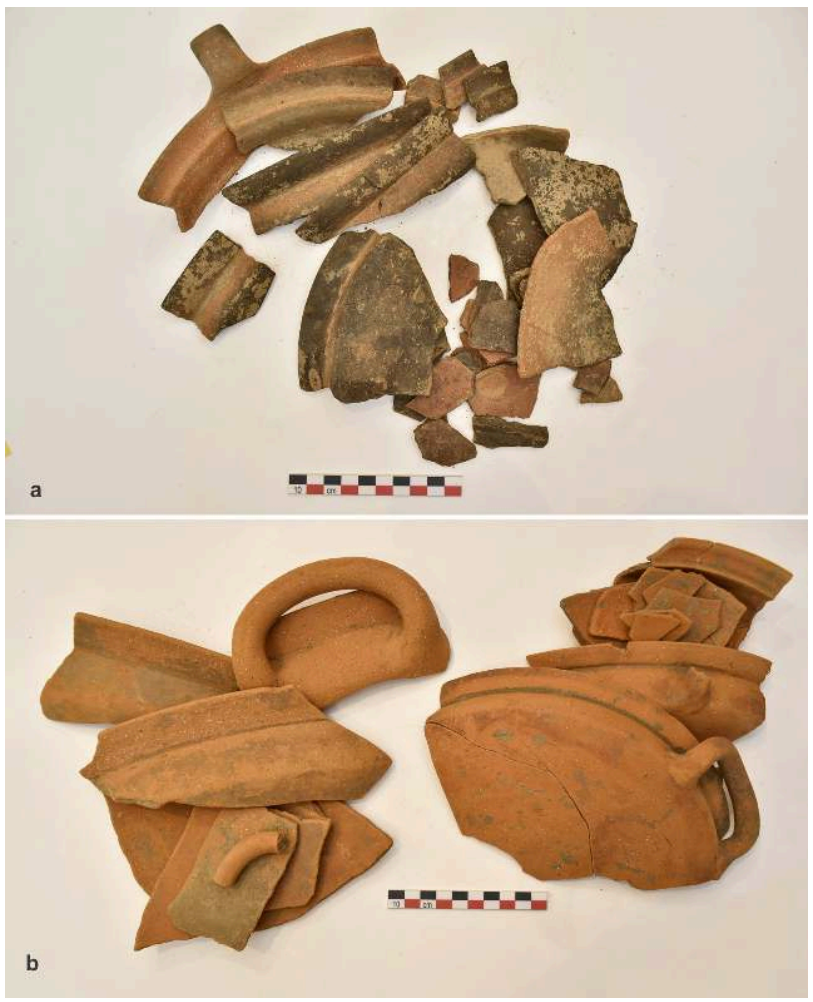

a. Lopas fragmentaire de la construction en schiste 37

b. Lopades fragmentaires de la construction en schiste 51.

a. Musée archéologie de Ténos, inv. ミ9231K7, b. Musée archéologique de Ténos, inv. ミ9208K1 et 三 9414. 
Fig. 6. Ténos, Xobourgo. Nécropole (parcelle Vardalakos).

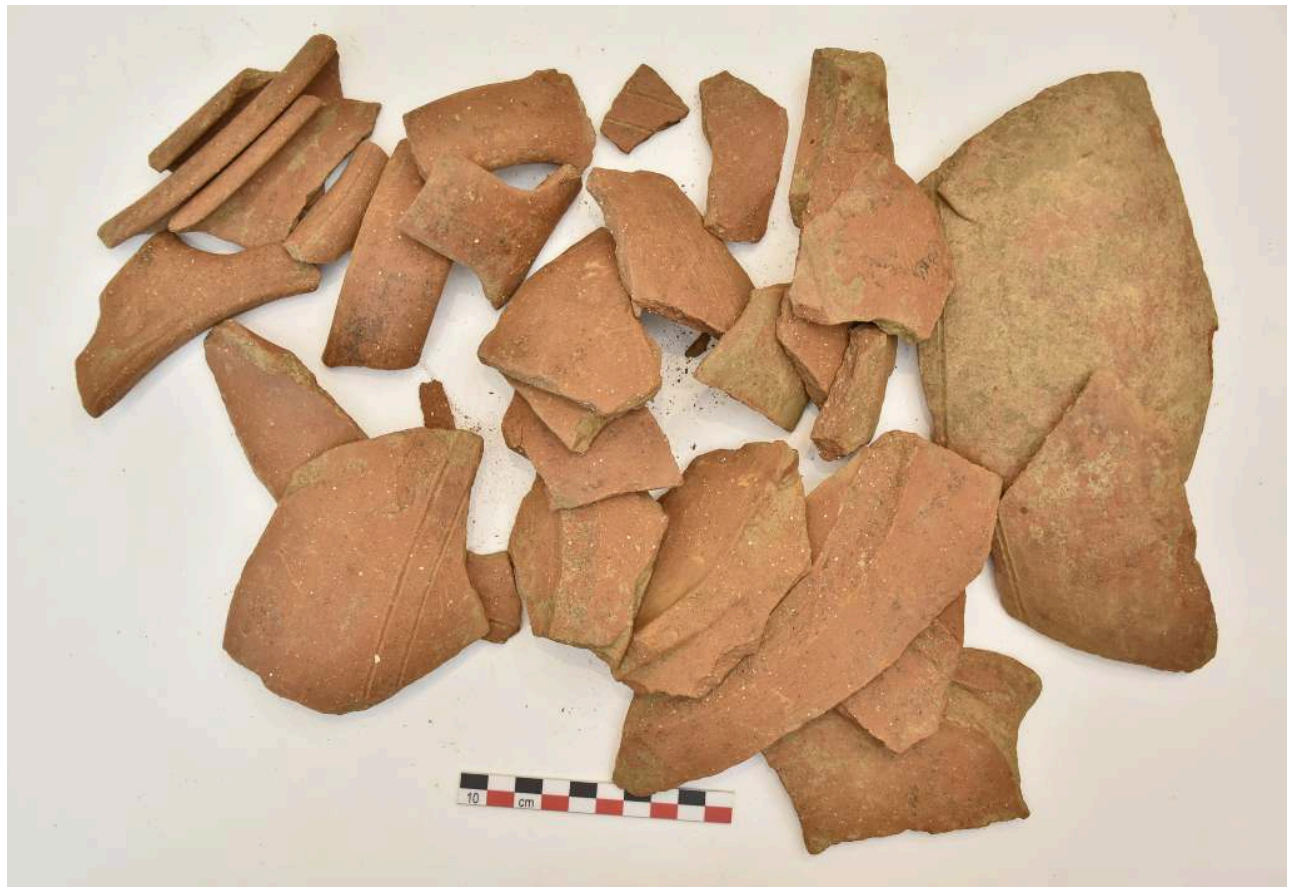

Chytra fragmentaire de la construction en schiste 37.

Musée archéologique de Ténos, inv. ミ9231K5.

Fig. 7. Ténos, Xobourgo. Nécropole (parcelle Vardalakos).

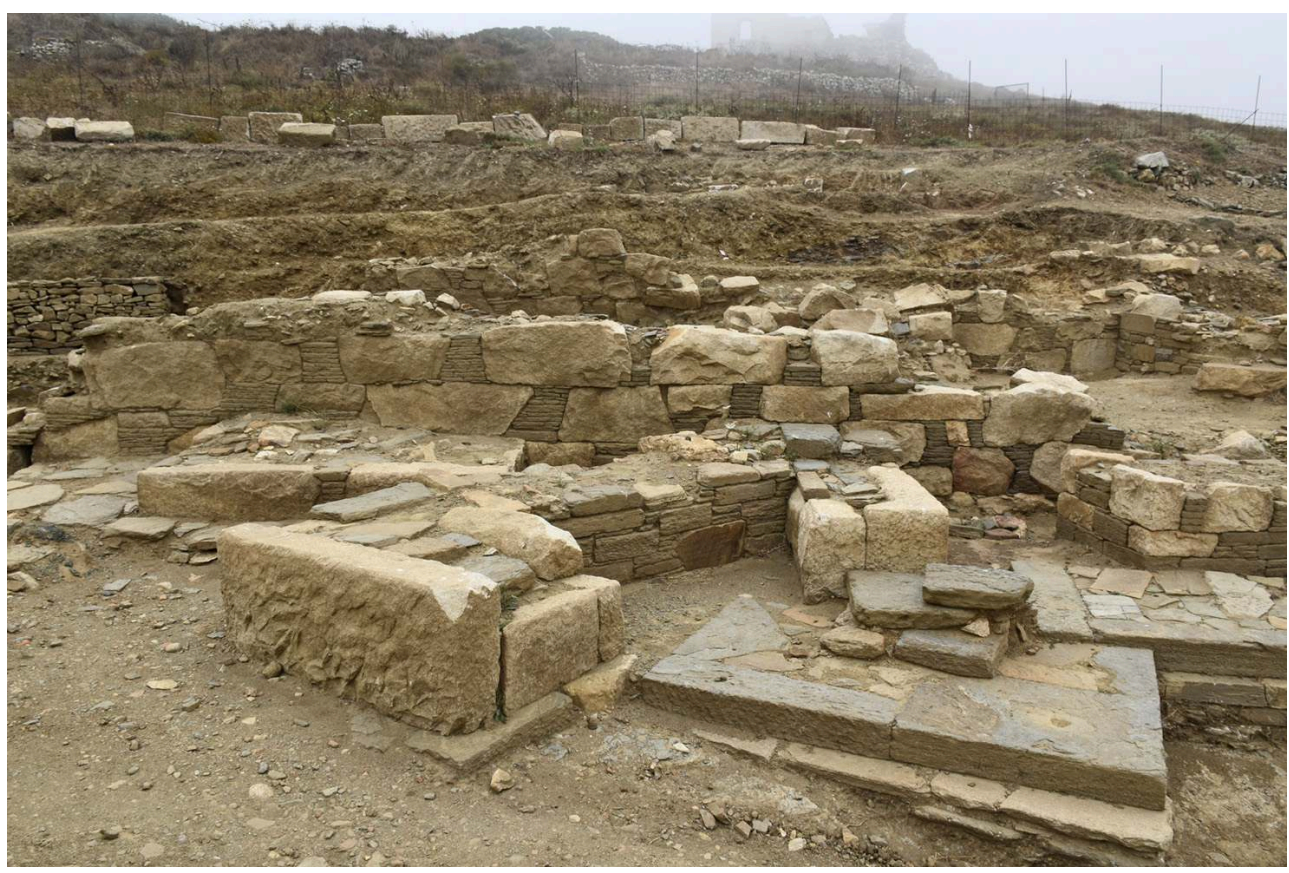

Cliché d'une partie des constructions en schiste devant le long bâtiment sur la zone nord de la nécropole.

6 Selon le matériel associé aux constructions en schiste 37 et 51 de la nécropole, nous pouvons constater que l'ensemble des récipients est presque exclusivement lié à la 
préparation et consommation des aliments, comme les marmites, les bassins, les supports et les lopades de tailles assez variantes avec ou sans couvercles. Même si les formes sont connues surtout par des contextes funéraires contemporaines attiques, c'est qui est unique à Ténos est la quantité de la céramique déposée et intentionnellement brisée sur et autour les constructions en schiste. En plus, la présence extrêmement faible dans les mêmes assemblages de la céramique fine et importée doit être signaler; ces vases qui ne dépassent pas 1,5\% dans les ensembles étudiés de la nécropole, semble davantage liés aux libations qu'à la consommation (fig. 8).

Fig. 8. Résultats des calculs qualitatifs et quantitatifs des assemblages céramiques provenant des constructions 51 et 37 de la nécropole (parcelle Vardalakos), Ténos, Xobourgo.

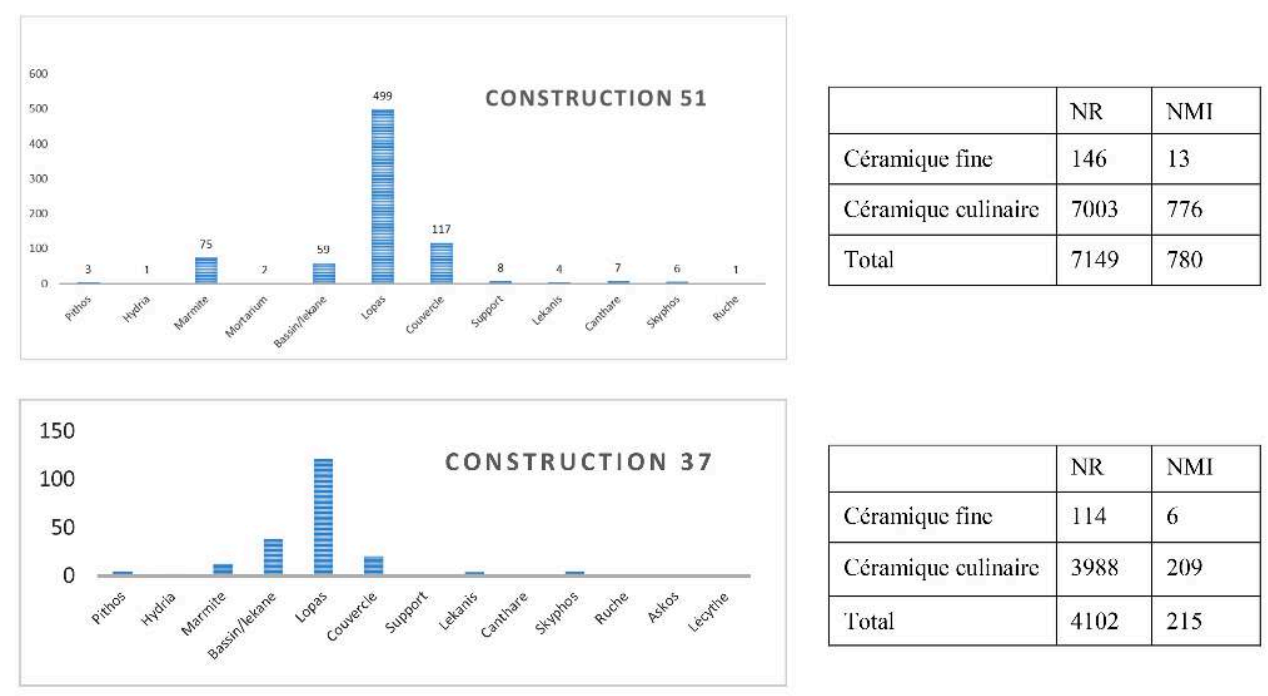

7 La plus grande partie de la céramique analysée peut être associée à une production locale, comme celle qui a été décrite pour les phases antérieures (géométrique et archaïque) par J.-S. Gros ${ }^{6}$. Il est donc possible d'intégrer la production céramique datant de la fin $v^{e}$ et au IV siècle av. J.-Chr., au sein d'une tradition céramique locale à partir déjà la période Géométrique ( $\mathrm{IX}^{\mathrm{e}}$-VIII ${ }^{\mathrm{e}}$ siècle av. J.-Chr.) et jusqu'à l'abandon progressif du site à partir de la fin du IV siècle av. J.-C. Si on reprend l'argumentation de C. Costin sur le travail des ateliers céramique ${ }^{7}$, il semble que on peut distinguer à Xobourgo une continuité des techniques, des recettes et de fabrication, qui semble plutôt liée à un savoir faire des communautés installées à Xobourgo, et probablement dans des zones à proximité immédiate. À partir de la période classique une certaine homogénéité des formes et des techniques ont été signalés pour la céramique commune, liée plutôt à la fois au développement des circuits commerciaux mais aussi à la production de masse ou à la spécialisation des ateliers touchant plus spécifiquement la céramique culinaire. ${ }^{8} \mathrm{Le}$ cas de la céramique téniote offre une vue alternative, d'une tradition de fabrication qui a maintenu une identité locale. Les analyses pétrographiques qui ont été planifiées vont tester nos observations macroscopiques et microscopiques (fig. 9). En plus, une analyse est prévue comprenant des échantillons des sources argileuses modernes, identifiées sur différentes parties de l'ile. Comme l'installation des poteries modernes étaient attestée jusqu'aux années 1960 sur l'ile, on attend que les données de cette production 
moderne contribuent à une meilleure définition et description de la céramique téniote dans la longue durée.

Fig. 9. Clichés (avec Dino Lite) de la surface deux pots (inv. K $9037 \mathrm{~K} 3$ et $9531 \mathrm{~K} 1$ ) illustrant les variations entre les différentes parties (intérieur - extérieur et corps - base).
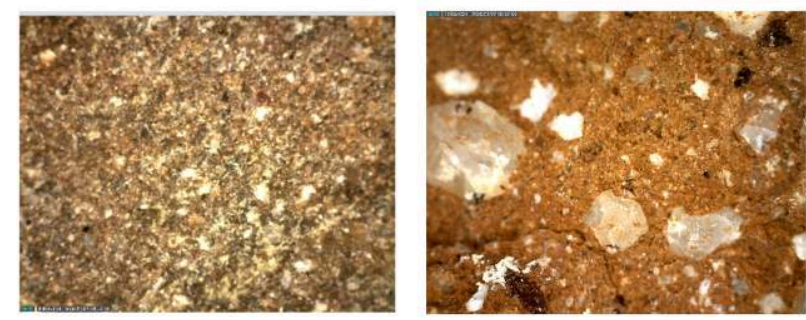

Inv. K 9037K3 (Mortarium)
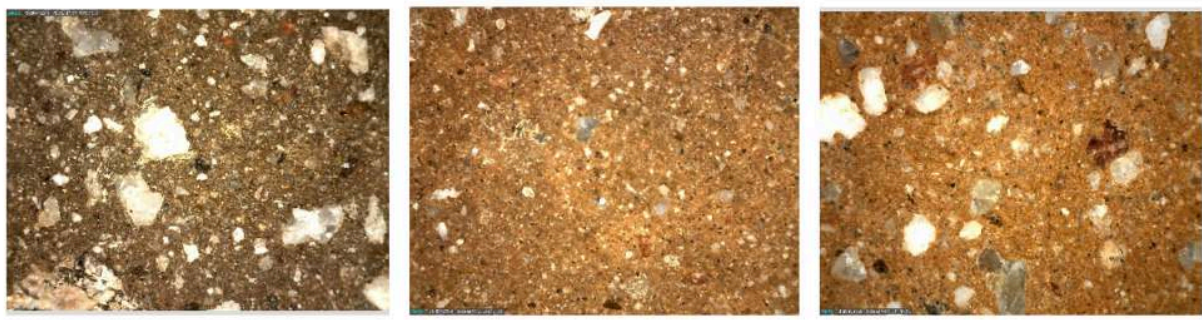

Inv. K 9531K1 (Bassin)

Photo de l'auteur.

\section{BIBLIOGRAPHIE}

BRøNS 2015

Cecile Brøns, « Textiles and Temple Inventories: Detecting an Invisible Votive Tradition in Greek Sanctuaries in the Second Half of the First Millenium BC », in Jane Fejfer, Mette Moltesen, Annette Rathje (éd.), Tradition. Transmission of Culture in the Ancient World, Copenhague, UP, Acta Hyperborea 14, 2015, p. 43-83.

BRøNS 2017

Cecile Brøns, Gods and garments: textiles in Greek sanctuaries in the 7th to the 1st centuries BC, Oxford, Oxbow Books, Ancient Textiles series 28, 2017.

COSTIN 2001

Cathy Lynne Costin, « Craft production systems », in Gary M. Feinman, T. Douglas Price (éd.), Archaeology at the Millenium. A sourcebook, New York, Springer, 2001, p. 273-327.

ÉTIENNE, KOUROU, SIMANTONI-BOURNIA 2013

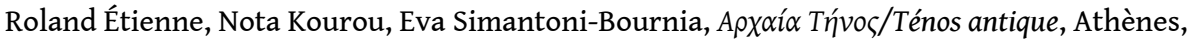

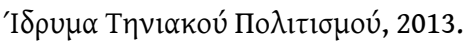


FERRARA, MEO 2017

Bianca Ferrara, Francesco Meo, « Loom weights in sacred contexts: The Square Building of the Heraion near the Sele River ", in Cecile Brøns, Marie-Louise Nosch (éd.), Textiles and cult in the ancient Mediterranean, Oxford, Oxbows Books, Ancient textiles series 31, 2017, p. 112-125.

GAMBACURTA 2017

Giovanna Gambacurta, « A loom for the Goddess: Tools for spinning and weaving from the sanctuary of the Goddess Reitia in Este (Padua) », Origini 40, 2017, p. 211-226.

GRECO 1997

Giovanna Greco, « Des étoffes pour Héra », in Julierre de La Genièvre (éd.), Hera. Images, espaces, cultes, Naples, Centre Jean Bérard, p. 185-199.

GROS 2015

Jean-Sébastien GROS, « Defining a Workshop for the Production of Domestic Pottery: the Case of Xobourgo on Tenos », dans Vicky Vlachou (éd.), Pots, Workshops and Early Iron Age Society: Function and Role of Ceramics in Early Greece, Bruxlles, ULB, Études d'archéologie 8, 2015, p. 75-79.

GROS 2017

Jean-Sébastien GROS, «Coarse, plain and cooking ware: seventh-century innovation for old-fashioned pots ", in Xenia Charalambidou et Catherine Morgan (éd.), Interpreting the seventh century BC: tradition and innovation, Oxford, Archaeopress Archaeology, 2017, p. 93-99.

KOUROU 2002

Nota Kourou, « Tenos-Xobourgo. From a refuge place to an extensive fortified settlement », in Maria Stamatopoulou, Marina Yeroulanou (éd.), Excavating Classical Culture. Recent archaeological discoveries in Greece, Oxford, Archeopress, BAR IS 1031, p. 255-268.

KOUROU 2019

Nota Kourou, " The classical cemetery of Xobourgo at Tenos. Between tradition and modernity ", in Heide Frielinghaus, Jutta Stroszeck, Panos Valavanis (éd.), Griechische Nekropolen: neue Forschungen und Funde, Möhnesee, Bibliopolis, 2019, p. 89-99.

MEYERS 2013

Gretchen E. Meyers, « Women and the production of ceremonial textiles: a reevaluation of ceramic textile tools in Etrusco-Italic sanctuaries », American Journal of Archaeology 117, 2013, p. 247-274.

SAXKJÆR et al. 2017

Sine Grove Saxkjær, Jan Kindberg Jacobsen, Gloria Paola Mittica, « Building V and ritual textile production at Timpone della Motta », in Cecile Brøns, Marie-Louise Nosch (éd.), Textiles and cult in the ancient Mediterranean, Oxford, Oxbows Books, Ancient textiles series 31, 2017, p. 91-103.

SOFRONIEW 2011

Alexandra Sofroniew, « Women's work: the dedication of loom weights in the sanctuaries of southern Italy », Pallas. Revue d'études antiques 86, 2011, p. 191-209.

VLACHOU 2019

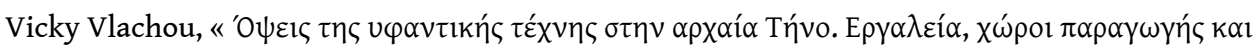

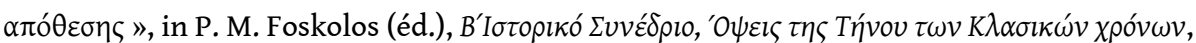
Ténos, 2019, p. 233-269.

VLACHOU 2020

Vicky Vlachou, « Les femmes et l'offrande d'étoffes dans les sanctuaires grecs. Une enquête sur la participation des femmes à la vie religieuse des cités archaïques et classiques », in Isabelle 
Algrain (éd.), Archéologie du genre. Construction sociale des identités et culture matérielle, Bruxelles, Université des Femmes, 2020, p. 111-143.

\section{NOTES}

1. KOUROU 2002 et 2019.

2. BRøNS 2015 ; BRøNS 2017, p. 36-37, p. 46-129.

3. VLACHOU 2019 et 2020.

4. SOFRONIEW 2011 ; MEYERS, 2013 ; GAMBACURTA 2017 ; SAXKJÆR et al., 2017 ; GRECO 1997 ; FERRARA, MEO 2017.

5. VLACHOU 2020.

6. GROS 2015.

7. COSTIN 2001.

8. GROS 2017.

\section{INDEX}

\section{Thèmes : EFA}

lieux https://ark.frantiq.fr/ark:/26678/pcrtYl3i34ZyI3

nature https://ark.frantiq.fr/ark:/26678/pcrtb1E0Dz7cSX

Année de l'opération : 2020

sujets https://ark.frantiq.fr/ark:/26678/pcrt1DMOWvDF4j, https://ark.frantiq.fr/ark:/26678/ pcrtfv4IrNiWE8, https://ark.frantiq.fr/ark:/26678/pcrtSPbJxwzVmX, https://ark.frantiq.fr/ark:/ 26678/pcrt05t5eGLZYZ, https://ark.frantiq.fr/ark:/26678/crtMK52omryzY, https:// ark.frantiq.fr/ark:/26678/pcrtzAt433wDSZ

\section{AUTEURS}

\section{VICKY VLACHOU}

École française d'Athènes, Académie royale des Sciences, des Lettres et des Beaux-Arts de Belgique, CReA-Patrimoine (ULB, Bruxelles) 